\title{
From Quercitols to Biologically Active Valienamine and Conduramine Derivatives: Development of Pharmacological Chaperones
}

\author{
Shinichi Kuno ${ }^{1}$; and Seiichiro Ogawa ${ }^{2}$ \\ ${ }^{1}$ Central Research Laboratories, Hokko Chemical Industry, Co., Ltd., 2165 Toda, Atsugi-shi, Kanagawa 243-0023, Japan \\ ${ }^{2}$ Department of Biosciences and Informatics, Faculty of Science and Technology, Keio University, \\ 3-14-1 Hiyoshi, Kohoku-ku, Yokohama, 223-8522, Japan \\ FAX: +81-46-228-0164, E-mail: kuno-s@hokkochem.co.jp, sogawa379@ybb.ne.jp
}

(Received on February 28, 2015, accepted on May 15, 2015)

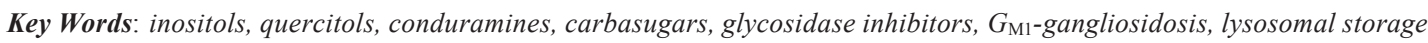
disorders, pharmacological chaperones

\begin{abstract}
Mutated glycosidases cannot be transported into the lysosome where they should function because the amino acid substitution causes misfolding of the enzymes. As a result, substrates such as glycolipids accumulate inside and outside the cell and cause severe symptoms. These diseases are known as lysosomal storage disorders (LSDs). Valid treatments for intractable LSDs are desired. Chaperone therapy is one of the novel approaches to remedy LSDs. This therapy uses low-molecular-weight molecules called pharmacological chaperones (PCs), which interact with the enzymes and render their folding correct. The authors have developed valienamine derivatives as PCs. The initial synthesis of these valienamine derivatives, however, required cumbersome chiral resolution. We therefore conducted convenient syntheses of the valienamine derivatives using chiral quercitols, where the stereochemical configurations of the hydroxy groups are similar to those of the valienamine derivatives. Meanwhile, many PCs have been designed based on glycosidase inhibitors. Those chaperone compounds need to strongly interact with the mutated enzymes and typically show inhibitory activity. Our valienamine derivatives also possess strong inhibitory activity. Such strong inhibitory action should be removed because PCs originally aim to increase the activity of the mutated enzymes. Therefore, we first simplified the main ring configuration from that of valienamine to that of conduramine to reduce the inhibitory activity. Next, to enhance the chaperone effect and increase the activity of the mutated enzyme, a structure-activity relationship study on the side chain of the conduramine derivatives was conducted. As a result, we found a novel PC for which the inhibitory activity was less than that of the seed compound while its chaperone effect was greater.
\end{abstract}

\section{A. Introduction}

Ogawa, one of the authors, has studied the synthesis and activity of carbasugars, where the oxygen atom in the pyranose ring is replaced by a carbon atom (1). The structure of carbasugars resembles that of real sugars, but carbasugars show diferente characters from sugars due to the replacement of oxygen with carbon in the ring. Among these compounds, valienamine derivatives resemble the six-membered oxocarbenium ion, the activated intermediate in the hydrolysis of glycosides, and show strong inhibition of glycosidases (2). Furthermore, interesting compounds that are effective for the treatment of LSDs were found from those valienamine derivatives (3). The synthesis of these carbasugars and valienamine derivatives was conducted using the racemic Diels-Alder adducts as starting materials, and optical resolution was necessary $(1,4,5)$.

Inositols are cyclohexanehexols, which have a hydroxy group on each of the six carbon atoms. Cyclohexanepentols, where one hydroxy group is removed from an inositol, are called quercitols. Some combinations of hydroxy configuration in inositols or quercitols are similar to those in sugars. These similarities in the stereochemical configuration of the hydroxy groups would be useful for the synthesis of carbasugars and valienamines. Moreover, quercitols are optically active.

In this minireview, the authors describe the convenient synthesis of valienamine derivatives using quercitols as starting materials and the development of a novel drug candidate for LSDs. Based on this, quercitols, valienamines, and conduramines as cyclitols/aminocyclitols, and genetically rare LSDs will be highlighted.

\section{B. Chemical and Microbial Synthesis of Quercitols}

There are nine isomers of inositols based on the stereochemistry of the hydroxy groups (6). Only two isomers, D-chiro-inositol and L-chiro-inositol, are optically active. The remaining are meso compounds. Each isomer has its own name, but myo-inositol may be the most important in the biochemistry field and the easiest inositol to obtain. For example, rice bran contains a certain amount of myo-inositol as phytic acid (7), which can be isolated.

Cyclohexanepentols, or deoxy-inositols, are called quercitols. There are 12 optical isomers among the 16 quercitol isomers (8), as the removal of one hydroxy group reduces their symmetry. Each 
isomer has its own name. The name "quercitol" is derived from the fact that it was first isolated from the acorns of Quercus (9). Some quercitols are isolated from natural sources (10), but there are reports that quercitols have been obtained by chemical synthesis $(10$, 11) and microbial fermentation (12).

In the chemical synthesis of quercitols, stereocontrol should be a key part. If one wants to obtain optically active quercitols from achiral starting materials, optical resolution or asymmetric synthesis will be necessary. In addition, appropriate protection of the hydroxy groups may be inevitable if one wants only a particular hydroxy group to react. Conversely, transformation of an achiral compound into a chiral compound and reaction of the particular functional group may be more easily conducted by microbial fermentation. Those are advantages to the fermentation method. Enzymes produced inside/outside the microorganism are responsible for conversion of the compounds. The active sites of enzymes are chiral and have the ability to transform a specific part of a compound in a chiral manner. Furthermore, without the isolation of enzymes, a reaction could be satisfactorily carried out by simply dissolving a precursor compound in the fermentation broth and ensuring that a suitable temperature is maintained. As a demerit of the fermentation method, it takes time for the detection, isolation and, if necessary, mutation and selection of the bacteria/ fungi that perform the desirable reaction. The authors' study group has been successful in obtaining three quercitols, (-)-vibo-quercitol, (+)-proto-quercitol, and (+)-epi-quercitol, by fermentation of myo-inositol (12).

\section{Chemical Synthesis of Valienamine Derivatives}

Valienamine is an unsaturated branched aminocyclitol, which is contained within the structures of a fungicide against rice sheath blight disease, validamycin A (1) (13), and a diabetes drug, acarbose (2) (14) (Fig. 1A). The structure of valienamine is similar to the six-membered oxocarbenium ion, the activated intermediate in the hydrolysis of sugars, and valienamine and its derivatives show inhibitory action against glycosidases $(2,4,5)$ (Fig. 1B).

As described above, the authors have synthesized several carbasugars and valienamine derivatives with furan (3) and acrylic acid (4) as starting materials and the Diels-Alder product (5 5 ) as an intermediate (Fig. 2A). This Diels-Alder reaction advantageously produces the endo isomer as a crystal (15). With this endo adduct as a key intermediate, the synthesis of carbasugars (16) and valienamines (17-19) were accomplished. Furthermore, compounds where valienamine and ceramide are combined with each other with the intent to inhibit glycosyltransferase (20), compounds in which the side chain is simplified to an $n$-octyl group, $N$-octyl$\beta$-valienamine (NOV, 6) (4), and the 4-hydroxy epimer of NOV, $N$ octyl-4-epi- $\beta$-valienamine (NOEV, 7) (5), were synthesized (Fig. 2B). NOV and NOEV became interesting compounds showing a therapeutic effect toward LSDs (21 and 22, respectively).

Nevertheless, the synthetic routes with Diels-Alder products as starting materials required cumbersome and inefficient optical resolution procedures. Particularly, certain amounts of NOV and NOEV were desired for studies on the treatment of LSDs. To correspond with this demand, we developed concise synthetic routes to valienamine derivatives starting from quercitols. Furan and acrylic acid for the Diels-Alder reaction are, however, readily available and not expensive. It is attractive that only the endo adduct can be isolated as a crystal. The activity of both enantiomers can be advantageously measured according to racemic synthesis

(A)

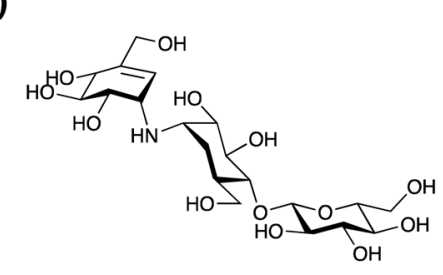

Validamycin A (1)

(B)

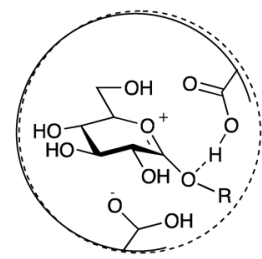

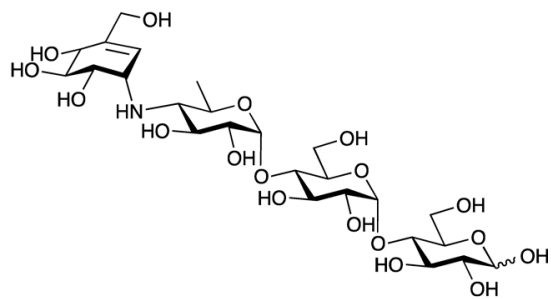

Acarbose (2)

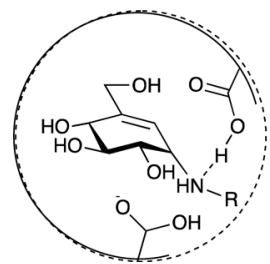

Fig. 1. (A) Chemical structures of validamycin A (1) and acarbose (2); (B) Structural similarity between the oxocarbenium intermediate in enzymatic hydrolysis of glucosides and valienamine derivatives. 
(A)

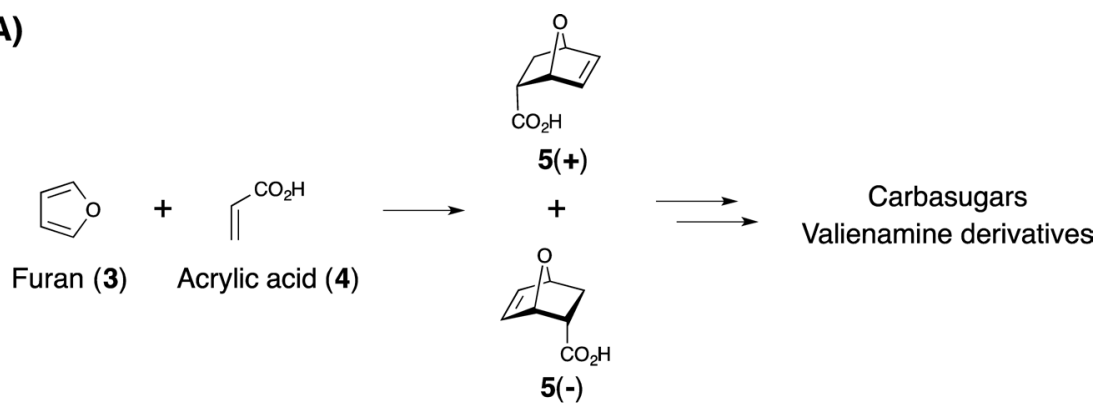

(B)

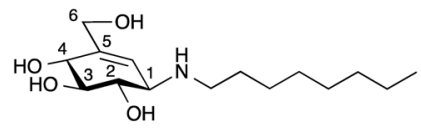

$N$-Octyl- $\beta$-valienamine (NOV, 6)

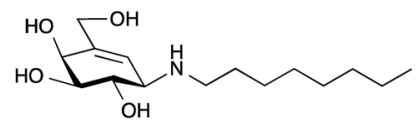

$N$-Octyl-4-epi- $\beta$-valienamine (NOEV, 7)

Fig. 2. (A) Diels-Alder adducts $(5 \pm$ ) as the key intermediates for the chemical synthesis of carbasugars; (B) Chemical structures of $N$ octyl- $\beta$-valienamine (NOV, 6) and $N$-octyl-4-epi- $\beta$-valienamine (NOEV, 7 ).

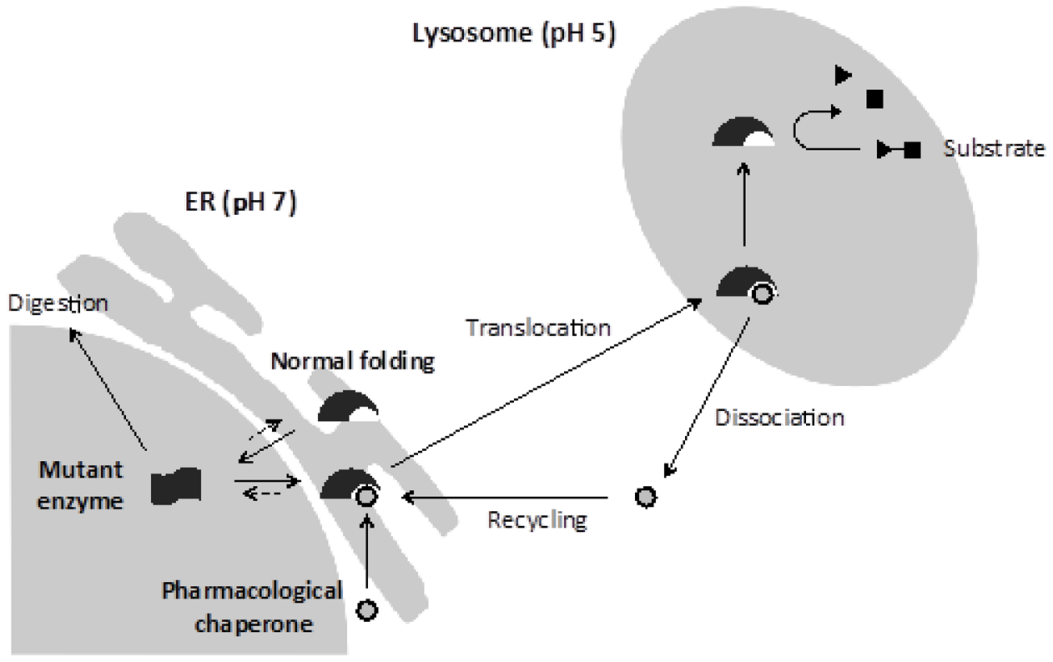

Fig. 3. Conceptual scheme for the mechanism of chaperone therapy.

and resolution method using an optical resolution column. Meanwhile, chiral starting materials are generally used for the development of biologically active carbohydrates. If the optically active adducts are produced by an asymmetric Diels-Alder reaction (23, 24) and other recent reactions, the former synthetic route would perform excellently.

\section{Lysosomal Storage Disorders (LSDs) and Pharma- cological Chaperones (PCs)}

LSDs are rare genetic diseases. Mutated glycosidases are not correctly folded and not delivered into the lysosome, which is their proper location to work. As a result, glycolipids and/or other substrates accumulate inside and outside the cell. There are as many LSDs as the number of mutated enzymes in the lysosome. LSDs are classified according to the accumulated substrates and the defi- cient enzymes. In addition, the condition and the onset of the disease differ based on which amino acid in the enzyme is substituted. The number of the patients with any LSD is not large. For example, Gaucher's disease, where the number of patients is relatively large among the LSDs, is caused by a mutation of $\beta$-glucosidase. Glucosylceramides, as substrates of the enzyme, are not degraded and accumulate inside and outside the cell. The prevalence rate is $2-2.1$ per 100,000 (25). In the pathogenesis of Fabry disease, where there is a deficiency in $\alpha$-galactosidase A, the number of the patients is relatively large, as was observed with Gaucher's disease. Globotriaosylceramides accumulate in Fabry cells. The prevalence is reported as $0.85-2.5$ per 100,000 . The authors have studied the development of the treatment for $\mathrm{G}_{\mathrm{M} 1}$-gangliosidosis, for which the pathogenesis is mutated $\beta$-galactosidase. $\mathrm{G}_{\mathrm{M} 1}$ gangliosides do not decompose and accumulate as the name of the 
disease indicates. Its prevalence rate is $0.33-1$ per 100,000 .

Two methods are known and used practically for the treatment of LSDs: substrate reduction therapy (26) and enzyme replacement therapy (27). Substrate reduction therapy focuses on decreasing the amount of unnecessary accumulated substrates, such as with glycolipids, where inhibitors of glycosyltransferases are administered. Miglustat ( $N$-butyl-deoxynojirimycin, 28) and eliglustat (29), consisting of the interesting ceramide-mimicking structure, are approved by the FDA in the United States. Meanwhile, many recombinant hydrolysis enzymes are produced by cultured cells for enzyme replacement therapy. This therapy is available for some LSDs; however, it is noted that the replacement enzyme does not work in the central nervous system due to not penetrating the blood brain barrier (27). There are quite a few LSDs that are not covered by these two therapies at the time of writing this minireview.

Alternative methods have been investigated for the treatment of LSDs. One of these is chaperone therapy with low molecular weight drugs. This therapeutic method is based on the idea that molecules called pharmacological chaperones (PCs) can stabilize the three-dimensional structure of the mutated enzymes, resulting in their translocation into the lysosome and expression of their enzyme activity (Fig. 3). The mechanism and history of chaperone therapy and PCs for LSDs were recently reviewed, for example, by Suzuki (30) and Boyd et al. (25). Although there are many reports regarding chaperone therapy, drugs based on this mechanism of action have not been used in clinical practice. Some PCs are in the preclinical development stage and others are in the research and development phase. Low-molecular-weight molecules, however, are able to pass through the blood brain barrier and treat the central nervous system symptoms. In addition, the combination of PCs and enzyme replacement therapy has been studied for the practical application of chaperone therapy (31).

\section{E. Concise Synthesis of Valienamine Derivatives Start- ing from Quercitols}

A convenient synthesis of $\operatorname{NOV}(6)$ and $\operatorname{NOEV~(7)~as~drug~}$ candidates for the treatment of LSDs was desired because the former synthetic route starting from a Diels-Alder product required optical resolution. Optically active quercitols appeared to be good starting material. In addition, there are some quercitols, in which the conformations of the hydroxy groups resemble those of sugars. For example, the configurations of the four hydroxy groups of (-)-vibo-quercitol (8) are identical to those of $\beta$-D-glucopyranose (9), and the configurations of the four hydroxy groups of $(+)$-proto-quercitol (10) are the same as those of $\beta$-D-galactopyranose (11) (Fig. 4A). The authors developed a convenient synthesis of NOV for Gaucher's disease from (-)-vibo-quercitol and a concise synthesis of NOEV for $\mathrm{G}_{\mathrm{Ml}}$-gangliosidosis from (+)-protoquercitol.

\section{E-1. Convenient Synthesis of NOV (32)}

First, the transformation of (-)-vibo-quercitol to (-)-2-deoxy-scyllo-inosose (12) was achieved by microbial conversion (Fig. 4B). In detail, Gluconobacter sp. AB10289 was cultivated in the appropriate medium, and after centrifugation, the

(A)
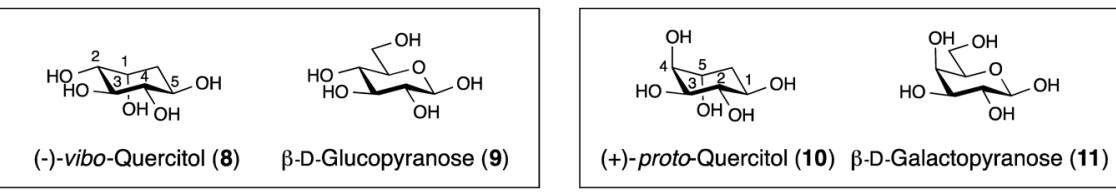

(B)

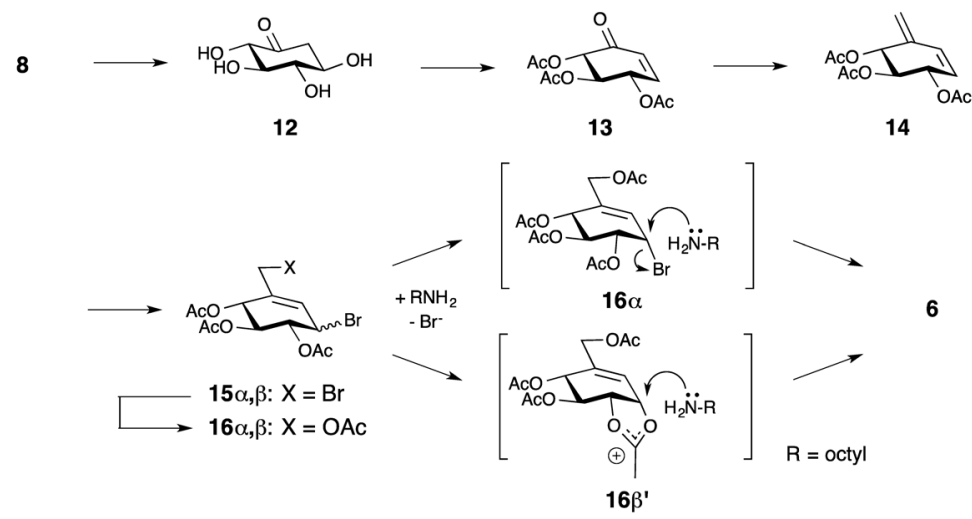

Fig. 4. (A) Structural similarity between (-)-vibo-quercitol (8) and $\beta$-D-glucopyranose (9), and (+)-proto-quercitol (10) and $\beta$-D-galactopyranose (11); (B) Synthesis of NOV (6) from (-)-vibo-quercitol (8). 
bacterial body was collected. To the residue was added an aqueous solution of (-)-vibo-quercitol. Incubation at a suitable temperature for one day quantitatively gave the compound $\mathbf{1 2}$ as the desired oxidation product. The purification of $\mathbf{1 2}$ was successfully carried out by simple filtration of the solution thorough cation and anion exchange resin columns.

Next, the compound $\mathbf{1 2}$ was acetylated using an acid catalyst followed by heating in acetic acid to give the unsaturated ketone (13). In this procedure, the acetyl groups have both protecting and leaving group properties and were useful for a concise synthesis. Methylenation of the compound $\mathbf{1 3}$ produced the conjugated diene (14), which had been versatile for the synthesis of carbasugars. Because the compound $\mathbf{1 3}$ was converted to a benzene scaffold under basic conditions, the compound $\mathbf{1 4}$ was obtained, even in low yield, under acidic conditions using the Nysted reagent. The synthesis after the compound $\mathbf{1 4}$ is the same as that using the Diels-Alder product as the starting material. Thus, after 1,4-dibromination of the diene (14), the primary bromide of the dibromo compound $(\mathbf{1 5} \boldsymbol{\alpha}, \boldsymbol{\beta})$ was treated with sodium acetate. The monobromo product $(\mathbf{1 6} \boldsymbol{\alpha}, \boldsymbol{\beta})$ was a mixture of stereoisomers; nevertheless, the reaction with octylamine gave solely the $\beta$-amino product (see also 33). The $\mathrm{S}_{\mathrm{N}} 2$ nucleophilic substitution of the $\alpha$-bromide (16 $\alpha$ ) with octylamine gave $\operatorname{NOV}(\mathbf{6})$, and with the $\beta$-bromide $(\mathbf{1 6} \boldsymbol{\beta})$, the amination producing NOV may occur via neighboring group participation of the 2-acetoxy group forming an oxocarbenium ion intermediate $\left(\mathbf{1 6} \boldsymbol{\beta}^{\prime}\right)$. In a synthetic scheme for NOEV discussed later, the corresponding $\alpha$-bromide and the epoxide from the corresponding $\beta$-bromide were isolated, and each compound was converted to NOEV with octylamine. The direct $\mathrm{S}_{\mathrm{N}} 2$ reaction of octylamine with the $\beta$-bromide (16ק) appeared not to have occurred due to steric hindrance, and the production of $\alpha$-valienamine derivatives was not observed. The acetyl functional groups contribute to the stereoselective amination here. Hence, the acetyl moieties in this synthetic scheme could be regarded as multifunctional groups. As described above, a convenient synthesis of NOV was developed from (-)-vibo-quercitol.

\section{E-2. Concise Synthesis of NOEV $(32,34)$}

NOEV is the 4-epimer of NOV. NOEV is a mimic of the activated intermediate in the hydrolysis of $\beta$-D-galactoside, in the same manner as NOV is a mimic of the intermediate in $\beta$-D-glucoside hydrolysis. The first synthesis of NOEV was performed by stereoinversion of the 4-hydroxy moiety by oxidation and reduction after proper protection of the other functional groups (5). A more convenient synthesis of NOEV was desired with the expectation of further studies for the treatment of $\mathrm{G}_{\mathrm{M} 1}$-gangliosidosis. We therefore started a concise synthesis of NOEV using the same strategy as that for NOV. $(+)$-proto-Quercitol, which is configurationally similar to $\beta$-D-galactopyranose, was used as the starting material.

First, the hydroxy groups, except the 5-hydroxy group of $(+)$-proto-quercitol $(\mathbf{1 0})$, were protected under acetal-forming conditions (35) (Fig. 5). Next, the unprotected 5-hydroxy moiety was oxidized (17), and the acetal-protected trans-hydroxy groups, more unstable in acid solution compared to the cis-acetal, were selectively deprotected under weakly acidic conditions. At the beginning, we planned acylation of the diol, formation of the unsaturated ketone, and methylenation, the same as in the synthesis of NOV. However, in fact, the diol was converted to the enol ester (18) with benzoyl chloride. The key intermediate conjugated diene (19) was fortunately obtained from the enol ester under Wittig reaction conditions. Methylenation of the enol ester moiety and the elimination of the benzoyloxy group at the $\beta$-position occurred in one step. The compound 19 corresponds to the galacto-type conjugated diene (14) in the NOV synthesis. Our previous experiences suggest that the $\beta$-position acetoxy/benzoyloxy group should be easily eliminated via an $\mathrm{E} 1 \mathrm{cB}$ reaction in acetylated/benzoylated inosose derivatives. Thus, one-step production of the compound $\mathbf{1 9}$ from the

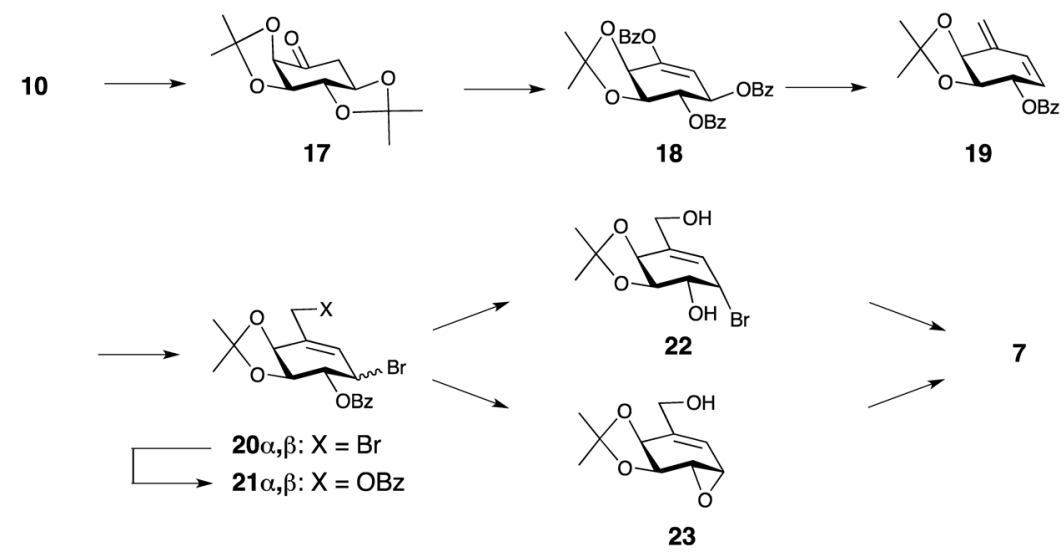

Fig. 5. Synthesis of NOEV (7) from (+)-proto-quercitol (10). 
enol ester (18) may have occurred by the mechanism below. First, the enolate was produced by attack of a phosphorous ylide on the enol ester moiety. Second, the benzoyloxy group at the $\beta$-position was eliminated via an $\mathrm{E} 1 \mathrm{cB}$ mechanism and the unsaturated ketone was generated. Finally, methylenation of the carbonyl group was afforded by the Wittig reaction. Following the same procedures as in the NOV synthesis yielded a mixture of dibromo $(\mathbf{2 0} \boldsymbol{\alpha}, \boldsymbol{\beta})$ and monobromo stereoisomers $(\mathbf{2 1} \boldsymbol{\alpha}, \boldsymbol{\beta})$. The benzoyl groups were deproteced under basic conditions and the $\alpha$-bromide (22) and the epoxide (23) were each isolated. Each compound produced NOEV (7) with octylamine; furthermore, a mixture of those compounds also gave solely NOEV. It should be considered effective that the isomer mixtures were able to be converted to only the targeted compound under identical reaction conditions. The isolation of the epoxide (23) confirmed that neighboring group participation was essential for the stereoselective reaction producing NOEV, the same as in the aforementioned synthesis of NOV. Moreover, the compound $\mathbf{2 3}$ showed good reactivity and regio- and stereoselectivity. This discovery was useful for the synthesis of $\mathrm{N}$-substituted conduramine derivatives. To summarize, NOV was conveniently synthesized from (-)-vibo-quercitol in seven steps and 3\% overall yield, and NOEV was concisely prepared from $(+)$-proto-quercitol in nine steps and $6 \%$ overall yield.

\section{F. Design and Synthesis of PCs}

PCs are considered to typically have interactions with the active center of mutated enzymes and stabilize their three-dimen- sional conformations. A low molecular weight molecule that fits into the active site/substrate binding site of the enzymes is a competitive inhibitor. Early PCs were found by observing that small amounts of some competitive inhibitors paradoxically enhanced the activity of mutated enzymes. Many pharmacological chaperone compounds were designed based on competitive inhibitors as seed compounds, and as a result, they themselves showed inhibitory activity. Examples of recent PCs are shown in Fig. 6. There are chaperone compounds based on a well-known glycosidase inhibitor, nojirimycin/1-deoxynojirimycin. A bicyclic nojirimycin derivative (24) (36) targets the treatment of Gaucher's disease. Among 1-deoxy-galacto-nojirimycin derivatives, the compound 25a (37) was found to remedy Fabry disease and the compound $\mathbf{2 5} \mathbf{b}(\mathbf{3 7}, 38)$ was effective to treat $\mathrm{G}_{\mathrm{Ml}}$-ganglosidosis. The side chain of compound 25a was modified to $\mathbf{2 6}$ for the treatment of Fabry disease recently (39). The L-iodonojirimycin derivative (27) showed chaperone activity in Gaucher cells (40). An isofagomine derivative (28), where one hydroxy moiety from 1-deoxynojirimycin is reduced, is focused on treating Gaucher's disease (41). Inosamine derivatives, such as bicyclic compounds (29) (42), compounds with an adamantyl moiety in the side chain (30) (43), and compounds with a long alkyl chain as the aglycone (31) (44), are reported as PCs. These inosamines aim to treat Gaucher's disease. Furthermore, it is reported that six-(32) (45) and seven-membered (33) (46) ring lactams showed chaperone activity in Gaucher cells. The development of as many PCs as possible may be preferable because the treatment effects by PCs vary due to the mutated amino acid positions,
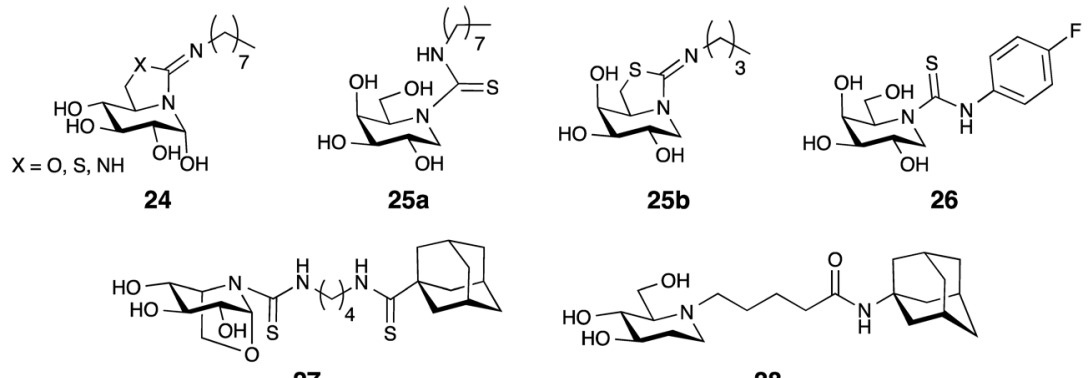

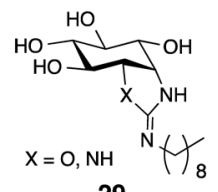

29

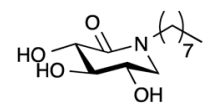

32

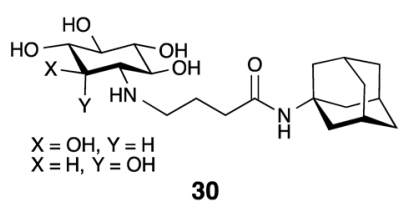

30

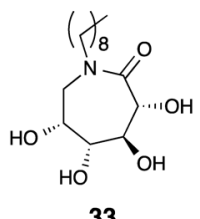

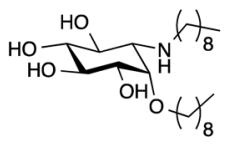

31

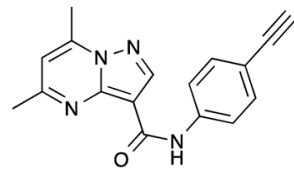

34

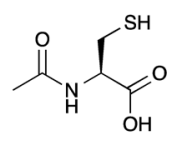

35

Fig. 6. Recent pharmacological chaperone compounds. 
even for the identical disease. In addition, as described above, many pharmacological chaperone compounds consist of a main ring mimicking the sugars, and a hydrophobic side chain. Considering that PCs with inhibitory activity are administered to LSD patients, strict control of dosage, and monitoring of pharmacokinetics should be essential. Recently, the search for PCs without inhibitory activity using high-throughput screening (34) (47) was reported. In addition, it was also reported that $N$-acetylcysteine (35) (48) bound to the allosteric site of mutated $\beta$-glucosidase and enhanced its enzyme activity. Intrinsically, PCs used for enhancing the mutated enzyme activity should not need inhibitory activity. Nevertheless, competitive inhibitor-type PCs are able to stabilize the mutated enzyme conformation, i.e., chaperone activity, by binding to the active site of the enzyme, i.e., competitive inhibition. Therefore, to merely weaken the inhibitory activity from PCs should require ingenuity.

The authors considered that modification of the structure of PCs could decrease their inhibitory activity while chaperone activity was maintained or even increased. Several compounds were prepared by modifying the structure of NOEV as a seed compound. Their inhibitory activity and chaperone activity were measured against normal human $\beta$-galactosidase and mutated human R201C $\beta$-galactosidase causing juvenile $\mathrm{G}_{\mathrm{M1}}$-gangliosidosis (49).

First, 6-deoxy-NOEV hydrochloride (36) and $\mathrm{N}$-octyl-conduramine F-4 hydrochloride (37), which are derivatives of NOEV hydrochloride (7a) without the hydroxy moiety and the hydroxymethyl moiety, respectively, were synthesized (Fig. 7A). It was expected that reducing the hydrogen bonding and hydrophobic interactions between the compounds and the enzyme should decrease the inhibitory activity. Removal of the hydroxymethyl group from the valienamine scaffold leads to the conduramines, which are amine derivatives of conduritol. Conduramines are named depending on the regio- and stereochemical configuration of the hydroxy and amino groups. There exists conduramine A to F, and numbering is followed, such as conduramine A-1 (11). Conduramines have not been isolated from natural sources and all of them are synthetic compounds. Some conduramine derivatives show glycosidase inhibitory activity (50).

For the synthesis of 6-deoxy-NOEV hydrochloride (36), the primary bromide moiety in the dibromo compound $(\mathbf{2 0} \boldsymbol{\alpha}, \boldsymbol{\beta})$ was selectively reduced under weakly reductive conditions, giving the $\alpha$-monobromide (38) after silica gel column purification (34) (Fig. 7B). Following debenzoylation, nucleophilic addition of octylamine, and deprotection of the acetal group gave the compound $\mathbf{3 6}$.

Synthesis of the $N$-substituted conduramine F-4 derivatives was conducted as given below (Fig. 7C). (+)-proto-Quercitol was protected by two isopropylidene groups (39), the same as in the first step of the NOEV synthesis, and the vacant 5-hydroxy group was mesylated (40). The cyclohexene (41) was obtained from the compound $\mathbf{4 0}$ by an E2 elimination reaction using a bulky base. Because a trans-isopropylidene is more easily deprotected than the cis form, selective deprotection of the trans-isopropylidene in the cyclohexene (41) was achieved with a weak acid, pyridinium $p$ toluenesulfonate (PPTS), yielding the diol (42). Using the Martin sulfurane (51), the epoxide (43) was obtained from the diol (42). The amine adducts (44) were regio- and stereoselectively obtained as target compounds in the conduramine synthesis in a fashion similar to the synthesis of NOEV.

One-carbon homologation is necessary for the synthesis of NOV and NOEV, for which the number of carbons in the main ring

(A)

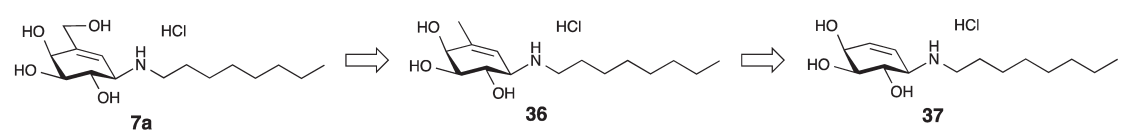

(B)

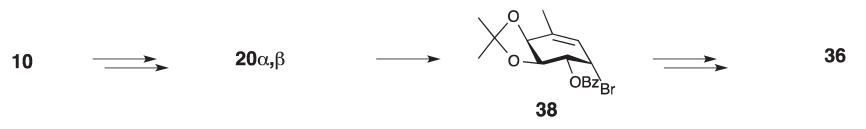

(C)

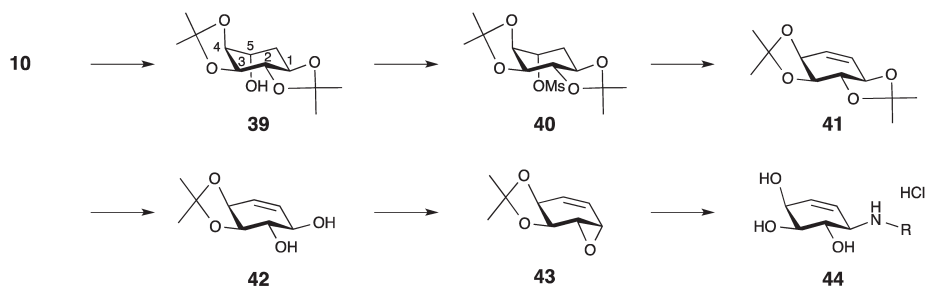

Fig. 7. (A) Design of 6-deoxy-NOEV hydrochloride (36) and N-octyl-conduramine F-4 hydrochloride (37) based on NOEV hydrochloride (7a); (B) Synthesis of 36 from (+)-proto-quercitol (10); (C) Synthesis of $N$-substituted conduramine F-4 derivatives (44) from 10. 
is seven, from quercitols, for which the number of carbons is six. Meanwhile, the number of carbons in the main ring of conduramine derivatives is six. Thus, the synthesis of $N$-substituted conduramine derivatives from quercitols does not need a carbon homologation reaction. Several conduramine derivatives with various side chains were successfully prepared. A structure-activity relationship study of $\beta$-galactosidase inhibitory and chaperone activity by these conduramine derivatives was conducted.

\section{G. Inhibitory and Mutated-enzyme Enhancement} Activity (Chaperone Activity) of Conduramine F-4 Derivatives (49)

The inhibitory activity of NOEV hydrochloride (7a), 6-deoxy NOEV hydrochloride (36), and $N$-octyl-conduramine F-4 hydrochloride (37) was reduced upon reduction of the hydroxymethyl moiety; the $\mathrm{IC}_{50}$ of 6-deoxy-NOEV (36) and $N$-octyl conduramine F-4 (37) was approximately $1 / 30$ and $1 / 70$ that of $\operatorname{NOEV~(7a),~re-~}$ spectively. The mutated-enzyme enhancement activity (chaperone activity) of NOEV (7a) peaked at a concentration of $2 \mu \mathrm{M}$; the mutated enzyme activity was increased 4.6-fold compared with no addition (Fig. 8). However, at a $20 \mu \mathrm{M}$ concentration, the mutated enzyme activity increased 3.6-fold. This suggested that the strong inhibitory activity of NOEV masked the chaperone activity at higher concentrations. The chaperone activity of 6-deoxy-NOEV (36) and $N$-octyl-conduramine F-4 (37), in contrast, increased along with the concentration within the tested concentrations. It was not masked at higher concentrations because the inhibitory activity of 6-dexoxy-NOEV and $N$-octyl-conduramine F-4 was reduced.

The inhibitory activity was successfully reduced by changing the valienamine scaffold to conduramine scaffold; next, we tried to increase the chaperone activity of the derivatives. It is known that there exists a hydrophobic region in the "adjacent" area near the active center of $\beta$-galactosidase (52). The hydrophobic side chain of the PCs could interact with this area in the enzyme. If the

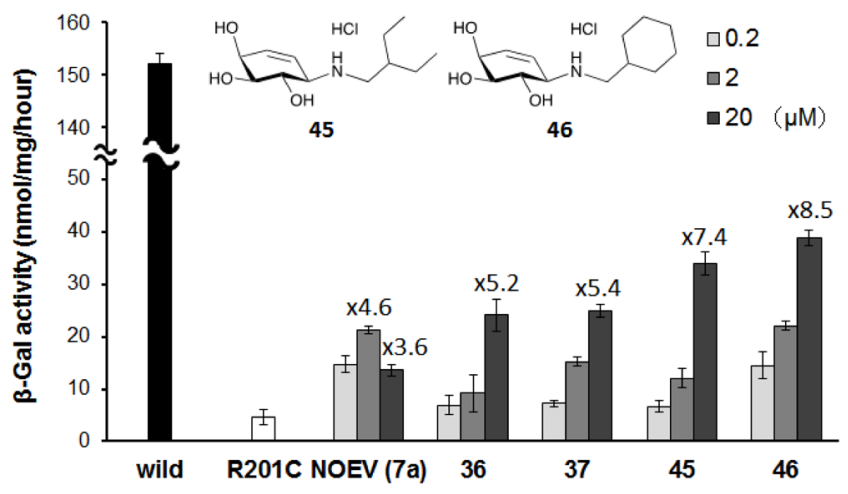

Fig. 8. Chaperone activity of $N$-substituted conduramine F-4 derivatives. interaction between the PCs and the mutated enzymes occurred at a site other than the active center, the three-dimensional structure of the mutated enzyme could be stabilized by the PCs without strong inhibitory activity. Based on this hypothesis, several $N$-substituted conduramine F-4 derivatives with hydrophobic side chains were synthesized and their chaperone activity was measured against R201C mutated $\beta$-galactosidase.

As a result, an $\mathrm{N}-\mathrm{CH}_{2}$-branched alkyl side chain was effective at increasing chaperone activity. In particular, a derivative with a 2-ethylbutylamino group (45) showed a 7.4-fold increase at $20 \mu \mathrm{M}$ and a derivative with a cyclohexylmethylamino group (46), which fixes the side chain conformation by forming a ring, showed the highest increase of 8.5 -fold at $20 \mu \mathrm{M}$ among the tested compounds. Interestingly, derivatives with an alkyl chain branched near the amine showed very low inhibitory and chaperone activities. The inhibitory activity of the 2-ethylbutyl derivative (45) and the cyclohexylmethyl derivative (46) were approximately 1/10 and $1 / 30$ that of $\operatorname{NOEV}(\mathbf{7 a})$, respectively. Their chaperone activity increased along with the concentrations until $20 \mu \mathrm{M}$, the highest concentration in the test series. Amphiphilic molecules typically show cytotoxicity. A conduramine F-4 derivative with decylamine, in fact, was toxic to the cells, while the compounds $\mathbf{4 5}$ and $\mathbf{4 6}$ did not show noticeable cytotoxicity. The compound 46 exhibited low inhibitory activity and remarkable chaperone activity at $20 \mu \mathrm{M}$. Furthermore, its chaperone activity at $0.2 \mu \mathrm{M}$ and $2 \mu \mathrm{M}$ was in the same range of that for the NOEV hydrochloride. This compound should be considered a second-generation PC following NOEV.

\section{H. Conclusion and Perspectives}

This review described a convenient synthesis of NOV for the treatment of Gaucher's disease, and NOEV for $\mathrm{G}_{\mathrm{M} 1}$-gangliosidosis, from quercitols as starting materials. In addition, this paper also described the design, synthesis, and bioactivity of novel drug candidates, for which the inhibitory action was reduced while the chaperone activity was increased, based on NOEV as a seed compound. We found that an $N$-cyclohexylmethyl conduramine F-4 derivative showed approximately $1 / 30$ the inhibitory activity and approximately twice the chaperone activity of NOEV hydrochloride. Further biochemical research on this compound should prove its remarkable potential as a pharmacological chaperone.

PCs reported so far typically consist of a main ring as a mimic of sugars and a hydrophobic side chain such as long alkyl chains. We suggested a strategy here: 1 . Decreasing the inhibitory activity of PCs by simplification of the main ring. 2. Increasing the chaperone activity by optimization of the side chain. Hopefully, this strategy can be applied to the structural modification of other PCs. Reports on the development of novel PCs appear to be increasing 
recently, and the appearance of more new compounds should be preferable because efficacy spectrum of PCs differs by the mutated amino acid positions. Much more attentions on rare LSDs will certainly help to develop effective therapeutic agents for the diseases.

\section{Acknowledgments}

We are thankful to Prof. Dr. E. Nanba and Prof. Dr. K. Higaki for conducting the biological assay and for valuable discussions. We are thankful to Dr. Y. Umezawa and Dr. R. Sawa for measuring the HRMS, and Dr. A. Takahashi and Mr. M. Yamaguchi for their support and useful suggestions.

\section{References}

1. Ogawa, S. (2004) Trends Glycosci. Glycotechnol. 16, 33-53.

2. Borges de Melo, E., da Silveira Gomes, A., and Carvalho, I. (2006) Tetrahedron 62, 10277-10302.

3. Suzuki, Y., Ogawa, S., and Sakakibara, Y. (2009) Perspect. Med. Chem. 3, 7-19.

4. Ogawa, S., Ashiura, M., Uchida, C., Watanabe, S., Yamazaki, C., Yamagishi, K., and Inokuchi, J. (1996) Bioorg. Med. Chem. Lett. 6, 929-932.

5. Ogawa, S., Matsunaga, Y. K., and Suzuki, Y. (2002) Bioorg. Med. Chem. 10, 1967-1972.

6. Loewus, F. A. (1990) Cyclitols. Methods In Plant Biochemistry, Vol. 2. Carbohydrates (Dey, P. M., and Harborne, J. B., eds.) pp. 219-233, Academic Press Inc, London.

7. Kasim, A. B., and Edwards, H. M. (1998) J. Sci. Food Agric. 76, 1-9.

8. McCasland, G. E., Furuta, S., Johnson, L. F., and Shoolery, J. N. (1961) J. Am. Chem. Soc. 83, $2335-2343$.

9. McCasland, G. E., Naumann, M. O., and Durham, L. J. (1967) Carbohydr. Res. 4, 516-518.

10. Aydin, G., Savran, T., Aktas, F., Baran, A., and Balci, M. (2013) Org. Biomol. Chem. 11, 1511-1524.

11. Duchek, J., Adams, D. R., and Hudlicky, T. (2011) Chem. Rev. 111, 4223-4258.

12. Takahashi, A., Kanbe, K., Tamamura, T., and Sato, K. (1999) Anticancer Res. 19, 3807.

13. Iwasa, T., Yamamoto, H., and Shibata, M. (1970) J. Antibiot. 23, 595-602.

14. Schmidt, D. D., Frommer, W., Junge, B., Muller, L., Wingender, W., Truscheit, E., and Shafer, D. (1977) Naturwissenschafter 64, 535-536.

15. Suami, T., Ogawa, S., Nakamoto, K., and Kasahara, I. (1977) Carbohydr. Res. 58, 240-244.

16. Ogawa, S., Iwasawa, Y., Nose, T., Suami, T., Ohba, S., Ito, M., and Saito, Y. (1985) J. Chem. Soc., Perkin Trans. 1, $903-906$.

17. Toyokuni, T., Ogawa, S., and Suami, T. (1983) Bull. Chem. Soc. Jpn. 56, 1161-1170.

18. Shibata, Y., and Ogawa, S. (1989) Carbohydr. Res. 189, 309-322.

19. Miyamoto, Y., and Ogawa, S. (1989) J. Chem. Soc., Perkin Trans 1, 1013-1018.

20. Tsunoda, H., Inokuchi, J., Yamagishi, K., and Ogawa, S. (1995) Liebigs Ann. Chem. 1995, $279-284$.

21. Lei, K., Ninomiya, H., Suzuki, M., Inoue, T., Sawa, M., Iida, M., Ida, H., Eto, Y., Ogawa, S., Ohno, K., and Suzuki, Y. (2007) Biochim. Biophys. Acta 1772, 587-596.

22. Matsuda, J., Suzuki, O., Oshima, A., Yamamoto, Y., Noguchi, A., Takimoto, K., Itoh, M., Matsuzaki, Y., Yasuda, Y., Ogawa, S., Sakata, Y., Nanba, E., Higaki, K., Ogawa, Y., Tominaga, L., Ohno, K., Iwasaki, H., Watanabe, H., Brady, R. O., and Suzuki, Y. (2003) Proc. Natl. Acad. Sci. U.S.A. 100, 15912-15917.

23. Ahrendt, K. A., Borths, C. J., and MacMillan, D. W. C. (2000) J. Am. Chem. Soc. 122, 4243-4244.

24. Payette, J. N., and Yamamoto, H. (2007) J. Am. Chem. Soc. 129, 9536-9537.

25. Boyd, R. E., Lee, G., Rybczynski, P., Benjamin, E. R., Khanna, R., Wustman, B. A., and Valenzano, K. J. (2013) J. Med. Chem. 56, $2705-2725$.

26. Platt, F. M., and Jeyakumar, M. (2008) Acta Paediatr. Suppl. 97(s457), 88-93.

27. Desnick, R. J., and Schuchman, E. H. (2002) Nat. Rev. Genet. 3, 954-966.

28. Ficicioglu, C. (2008) Ther. Clin. Risk Manag. 4, 425-431.

29. Shayman, J. A. (2010) Drugs Future 35, 613-620.

30. Suzuki, Y. (2014) Proc. Jpn. Acad. Ser. B 90, 145-162.

31. Benjamin, E. R., Khanna, R., Schilling, A., Flanagan, J. J., Pellegrino, L. J., Brignol, N., Lun, Y., Guillen, D., Ranes, B. E., Frascella, M., Soska, R., Feng, J., Dungan, L., Young, B., Lockhart, D. J., and Valenzano, K. J. (2012) Mol. Ther. 20, 717-726.

32. Kuno, S., Takahashi, A., and Ogawa, S. (2011) Bioorg. Med. Chem. Lett. 21, 7189-7192.

33. Ogawa, S., Sakata, Y., Ito, N., Watanabe, M., Kabayama, K., Itoh, M., and Korenaga, T. (2004) Bioorg. Med. Chem. 12, $995-1002$.

34. Kuno, S., Takahashi, A., and Ogawa, S. (2013) Carbohydr. Res. 368, 8-15.

35. Wacharasindhu, S., Worawalai, W., Rungprom, W., and Phuwapraisirisan, P. (2009) Tetrahedron Lett. 50, $2189-2192$.

36. Luan, Z., Higaki, K., Aguilar-Moncayo, M., Ninomiya, H., Ohno, K., García-Moreno, M. I., Ortiz Mellet, C., García Fernández, J. M., and Suzuki, Y. (2009) ChemBioChem 10, 2780-2792.

37. Aguilar-Moncayo, M., Takai, T., Higaki, K., Mena-Barragán, T., Hirano, Y., Yura, K., Li, L., Yu, Y., Ninomiya, H., García-moreno, M. I., Ishii, S., Sakakibara, Y., Ohno, K., Nanba, E., Ortiz Mellet, C., García Fernández, J. M., and Suzuki, Y. (2012) Chem. Commun. (Camb.) 48, $6514-6516$.

38. Takai, T., Higaki, K., Aguilar-Moncayo, M., Mena-Barragán, T., Hirano, Y., Yura, K., Yu, L., Ninomiya, H., García-moreno, M. I., Sakakibara, Y., Ohno, K., Nanba, E., Ortiz Mellet, C., García Fernández, J. M., and Suzuki, Y. (2013) Mol. Ther. 21, 526-532.

39. Yu, Y., Mena-Barragán, T., Higaki, K., Johnson, J. L., Drury, J. E., Lieberman, R. L., Nakasone, N., Ninomiya, H., Tsukimura, T., Sakuraba, H., Suzuki, Y., Nanba, E., Ortiz Mellet, C., García Fernández, J. M., and Ohno, K. (2014) ACS Chem. Biol. 9, $1460-1469$.

40. Alfonso, P., Andreu, V., Pino-Angeles, A., Moya-Garcia, A. A., García-moreno, M. I., Rodríguez-Rey, J. C., Sánchez-Jiménez, F., Pocoví, M., Ortiz Mellet, C., García Fernández, J. M., and Giraldo, P. (2013) ChemBioChem 14, 943-949. 
41. Yu, Z., Sawakar, A. R., Whalen, L. J., Wong, C.-H., and Kelly, J. W. (2007) J. Med. Chem. 50, 94-100.

42. Trapero, A., Alfonso, I., Butters, T. D., and Llebaria, A. (2011) J. Am. Chem. Soc. 133, 5474-5484.

43. Trapero, A., Egido-Gabás, M., and Llebaria, A. (2013) Med. Chem. Commun. 4, 1584-1589.

44. Trapero, A., González-Bulnes, P., Butters, T. D., and Llebaria, A. (2012) J. Med. Chem. 55, 4479-4488.

45. Wang, G.-N., Reinkensmeier, G., Zhang, S.-W., Zhou, J., Zhang, L.-R., Zhang, L.-H., Butters, T. D., and Ye, X.-S. (2009) J. Med. Chem. 52, 31463149 .

46. Wang, G.-N., Twigg, G., Butters, T. D., Zhang, S., Zhang, L., Zhang, L.-H., and Ye, X.-S. (2012) Org. Biomol. Chem. 10, $2923-2927$.

47. Patnaik, S., Zheng, W., Choi, J. H., Motabar, O., Southall, N., Westbroek, W., Lea, W. A., Velayati, A., Goldin, E., Sidransky, E., Leister, W., and Marugan, J. J. (2012) J. Med. Chem. 55, 5734-5748.

48. Porto, C., Ferrara, M. C., Meli, M., Acampora, E., Avolio, V., Rosa, M., Cobucci-Ponzano, B., Colombo, G., Moracci, M., Andria, G., and Parenti, G. (2012) Mol. Ther. 20, 2201-2211.

49. Kuno, S., Higaki, K., Takahashi, A., Nanba, E., and Ogawa, S. (2015) Med. Chem. Commun. 6, 306-310.

50. Łysek, R., Schütz, C., Favre, S., O’Sullivan, A. C., Pillonel, C., Krülle, T., Jung, P. M. J., Clotet-Codina, I., Esté, J. A., and Vogel, P. (2006) Bioorg. Med. Chem. 14, 6255-6282.

51. Martin, J. C., Franz, J. A., and Arhart, R. J. (1974) J. Am. Chem. Soc. 96, 4604-4611.

52. Suzuki, H., Ohto, U., Higaki, K., Mena-Barragán, T., Aguilar-Moncayo, M., Ortiz Mellet, C., Nanba, E., García Fernández, J. M., Suzuki, Y., and Shimizu, T. (2014) J. Biol. Chem. 289, 14560-14568.

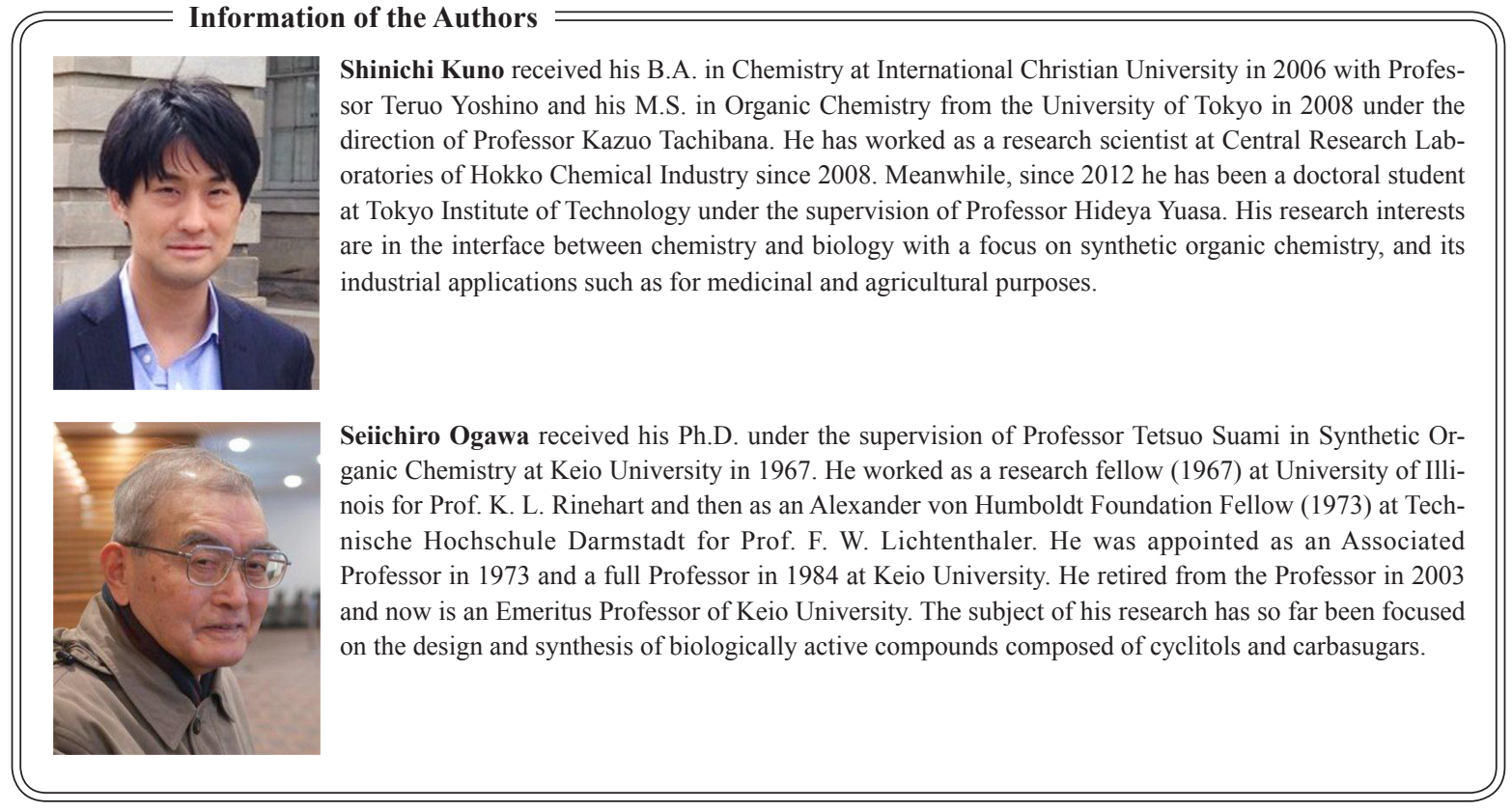

